\title{
Impacto ambiental de la Compañía minera Southern Perú Cooper Corporation en América Latina: Una aproximación histórica de comienzos del siglo $\mathrm{XX}$ a la actualidad
}

\author{
Environmental impact of the Southern Peru Copper \\ Corporation Mining Company in Latin America: A historical \\ approach from the Early 20th Century to the present
}

\author{
Sol Pérez-Jiménez ${ }^{1}$ \\ Universidad Nacional Autónoma de México, México
}

\begin{abstract}
Resumen
A pesar del reconocimiento de que fue a partir de la década de los 90 y la imposición de normativas neoliberales que se dio un aumento drástico de la extracción minera en América Latina, la Megaminería metálica en la región tiene sus antecedentes desde principios de silgo XX. En el presente artículo revisamos este fenómeno a través de la historia de una de las compañías mineras más importantes de la época, la American Mining and Smelting Company (Asarco), hoy subsidiaria de la compañía minera Grupo México. Que empezó a operar en Perú desde 1921 y, desde entonces, ha expandido su presencia en la región y se ha mantenido como uno de los principales productores de cobre a nivel mundial. En el trabajo se describe como todo ello ha implicado numerosos conflictos sociales y fuertes impactos ambientales reportados desde mediados del siglo XX, y perpetuados hasta la actualidad. Finalmente, se pone en duda si dichas prácticas empresariales e industriales deberían de seguirse reproduciendo en un contexto de alta conflictividad, escases de recursos y cambio climático.
\end{abstract}

Palabras clave: Minería, América Latina, Impacto Ambiental, Territorio, Grupo México.

1 Estudiante del Doctorado en Geografía, Universidad Nacional Autónoma de México, UNAM.

Email: solperezj@gmail.com

Este artículo corresponde a la ponencia presentada en el 35th Conference of Latin American Geographers realizada en San José, Costa Rica del 20 al 22 de mayo del 2018. 
Sol Pérez-Jiménez. Environmental impact of the Southern Peru Copper Corporation Mining Company in Latin America: A historical approach from the Early 20th Century to the present

\begin{abstract}
Despite the recognition that it was from the 90s and the imposition of neoliberal regulations that there was a drastic increase in mining extraction in Latin America, the transnational mining in the region has its antecedents since the beginning of XX century. The paper reviews this phenomenon through the history of one of the most important mining companies of the time, the American Mining and Smelting Company (Asarco), today a subsidiary of the mining company Grupo México. It started operating in Peru since 1921 and, since then, it has expanded its presence in the region and has remained one of the world's leading copper producers. The work describes how all this has involved numerous social conflicts and strong environmental impacts reported since the mid-twentieth century and perpetuated to this day. Finally, it is questioned whether these business and industrial practices should continue to be reproduced in a context of high environmental conflicts, lack of resources and climate change.
\end{abstract}

Keywords: Mining, Latin America, Environmental Impact, Territory, Grupo México

\title{
Introducción
}

El trabajo que se presenta a continuación caracteriza el proceso de expansión territorial de una corporación minera trasnacional, la empresa Grupo México. Tercer productor de cobre a nivel mundial. La cual ha operado a lo largo del continente americano desde principios del siglo XX. Cuando, como consecuencia de la revolución industrial y tecnológica, se dio una gran demanda de cobre a nivel mundial e inicio la explotación a gran escala de cobre, incluso de yacimientos de baja ley, accesibles entonces a las nuevas tecnologías de tajo abierto.

Es precisamente en ese contexto que se fundaron a principios del siglo XX las grandes corporaciones mineras en Estados Unidos, las cuales articulaban intereses económicos de los principales grupos empresariales. En 1901 se forma en Estados Unidos la empresa antecesora de Grupo México, la American Mining and Smelting Company (Asarco), hoy subsidiaria de dicho consorcio. En sus inicios Asarco estuvo ligada a importantes accionistas norteamericanos como la Phelps Dodge Overseas Capital Co., la Cerro de Pasco Co. y la Newmont Mining Company, que controlan el $20 \%$ de la fundición y el 15\% de la refinación del cobre en el mundo (Sánchez, 1988:111).

\section{Expansión de la compañía en Perú}

Asarco comenzó a operar en el norte de Perú en 1921 a través de su filial Nothern Peru Mining and Smelting Company. Año en que la compañía adquirió 73 concesiones mineras, con un total de 539 has. en el distrito 
Sol Pérez-Jiménez. Impacto ambiental de la Compañía minera Southern Perú Cooper Corporation en América Latina: Una aproximación histórica de comienzos del siglo XX a la actualidad

minero de Quiruvilca, provincia de Santiago de Chuco departamento de La Libertad. (Arciniega, 1986:14). Donde desarrolló la mina subterránea de cobre "Quiruvilca", la cual detuvo sus operaciones entre 1930 y 1936 debido a la caída del precio de los metales. La mina se fue ampliando al pasar de las décadas. En 1940 se construyó una planta concentradora de cobre de 300 ton/día. Tras sucesivas ampliaciones, para 1967 la planta llega a producir 1,100 ton/día. Ese año se implementaron circuitos de recuperación de plomo y zinc. En 1972 se instaló un sistema de recirculación de aguas ácidas para lixiviar minerales de cobre en la mina. En 1978 la compañía, en asociación a varios capitales peruanos, cambió su razón social por Corporación Minera North Perú S.A. A partir de esa fecha se efectúan importantes inversiones que permiten la ampliación de la producción llegando en 1980 a 1,650 ton/día (Rosario, 2014: 4). En el año 1995, la Corporación Minera Nor Perú S.A., luego de largo estancamiento en manos de la empresa norteamericana ASARCO, pasa a pertenecer a Pan American Silver Corporation, llegando a producir ese año 1,865 ton/día.

Para el segundo proyecto de Asarco en Perú la empresa constituyó en 1952 la filial Southern Peru Copper Corporation. La cual, como se narra a continuación, se convirtió en el principal productor de cobre de este país consolidándose desde sus inicios como una firma con un gran poder económico y político. En 1954 la Southern firmó un convenio con el Gobierno Peruano para la explotación del proyecto minero Toquepala, ubicado en el departamento de Tacna al Sur del País. Dicho convenio se ha considerado por múltiples analistas (Malpica, 1976; Arciniega, 1986) como radicalmente injusto. Pues, amparado en el Art. 56 del Código de Minería de 1950, considera al Yacimiento de Toquepala como marginal, logrando con ello la excepción de varios impuestos y demás beneficios fiscales. Entre 1956 y 1959 se llevó a cabo la construcción de la mina de Toquepala y toda la infraestructura necesaria para su operación, como la línea del ferrocarril, una fundición, muelle, campamentos, etc. (Arciniega, 1986:16). La mina empezó a extraer mineral desde 1960 llegando a las183,988 ton anuales.

La cuestión es que desde los inicios de Toquepala y la fundición de Ilo comenzó una serie de arbitrariedades e impactos al ambiente y la población local que se continúan perpetuando hoy en día. “Apenas meses después de inauguradas las operaciones de la empresa, los agricultores del 
Valle de Ilo denunciaron ante el Ministerio de Agricultura que los humos de la fundición estaban dañando sus diversos cultivos" (Días, 1988: 56).

En 1969, la empresa logró firmar un contrato con el gobierno militar de Velasco (1048 -1956) para la Mina de Cuajone, en el departamento de Tacna. Para llevar a cabo dicha operación la empresa obtuvo financiamiento de más de 50 bancos de cuatro continentes, la inversión total ascendió a 683 millones de dólares (Arciniega, 1986:24-25). La mina comenzó a construirse en 1970 y empezó a operar en 1976, convirtiendo a la Southern en el principal productor de Cobre de Perú. Lo cual transformó completamente la estructura económica de las exportaciones peruanas, convirtiendo al cobre en el principal producto de exportación, y segundo generador de divisas, después del petróleo.

Implicaciones sociales y ambientales de la explotación minera de SPCC

El aumento de la producción de la empresa implicó también el incremento de su demanda de agua, como de sus residuos industriales (jales mineros). Para 1980 ambas minas demandaban 1,500 litros de agua por segundo, actualmente la demanda es de 1950 litros. La compañía posee 9 licencias de uso de aguas que fueron aprobadas hace 56 y 41 años para extraer el recurso hídrico de las cabeceras de cuencas de esa región, para su mina en Toquepala (Tacna) y luego en Cuajone (Moquegua). Sus licencias le permiten extraer, tanto aguas superficiales, como subterráneas. 
Sol Pérez-Jiménez. Impacto ambiental de la Compañía minera Southern Perú Cooper Corporation en América Latina: Una aproximación histórica de comienzos del siglo XX a la actualidad

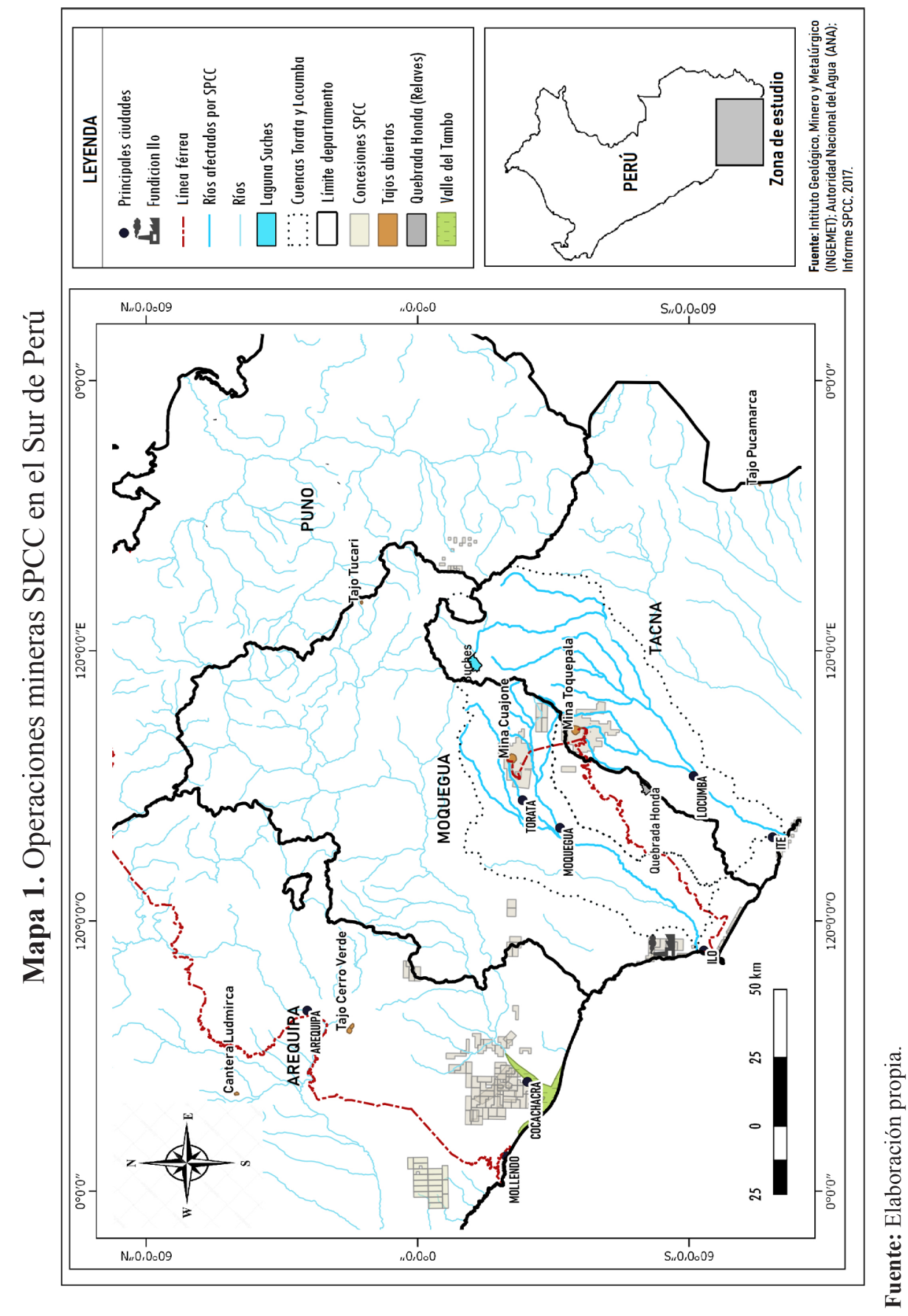


Con el paso del tiempo, la utilización de agua de pozos impactó en las cuencas y en los bofedales. Un ejemplo de este efecto son los bofedales moqueguanos de Titijones, que prácticamente han desaparecido y ahora son extensas zonas resecas. Empezaron a desaparecer debido a la sobre explotación de la laguna de Suches, un espejo de agua limítrofe entre Moquegua y Tacna. Peor aún, esta laguna hace mucho tiempo que se habría secado sino fuera porque la minera excavó los pozos con los que mantienen artificialmente el volumen de agua de esta laguna. Aun así, la merma en su nivel de agua es evidente, pero la minera sigue utilizando este recurso para los procesos en ambas minas de cobre (Informe LABOR, 2015).

Respecto al tema de los relaves mineros, estamos hablando de una descarga anual de 40 millones de metros cúbicos de desechos con altos contenidos de silicatos, metales pesados, cianuro y otros agentes tóxicos que, hasta 1996, se arrojaron directamente al mar a través de $21 \mathrm{Km}$ del Río Locumba, desembocando en la bahía de Ite. Donde se ha reportado que aproximadamente $300 \mathrm{Km} 2$ de la bahía quedaron contaminados, pues dichos relaves se fueron acumulando hasta ampliar el perímetro costero, ocasionando impactos ambientales graves, especialmente en la fauna marina, viéndose afectada con ello la pesca local. 
Sol Pérez-Jiménez. Impacto ambiental de la Compañía minera Southern Perú Cooper Corporation en América Latina: Una aproximación histórica de comienzos del siglo XX a la actualidad

Figura 1. Relaves mineros vertidos en Ite hasta 1996
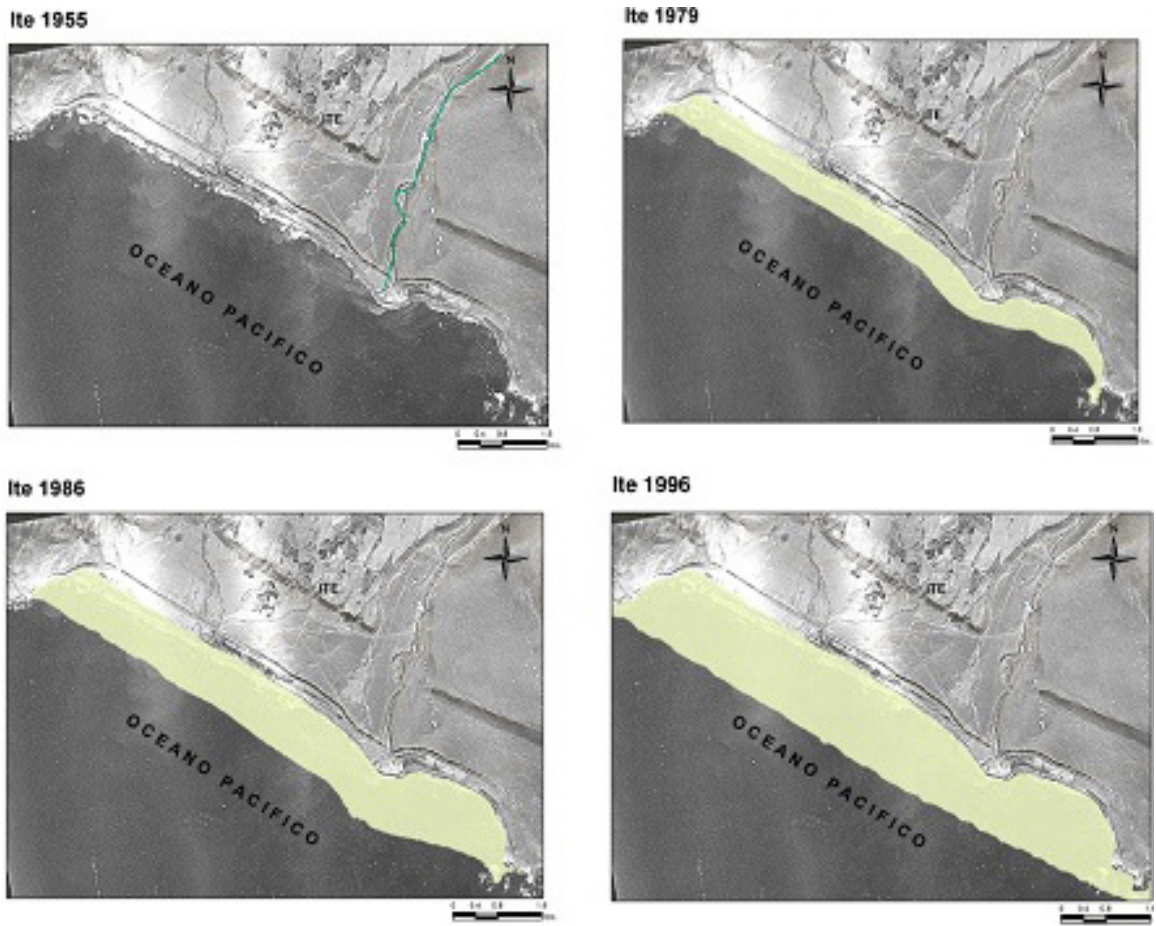

Fuente: SPCC, 2014.

A partir de 1996, la empresa empezó a verter sus relaves en un predio denominado Quebrada Honda, ver mapa 1. En 2010 la Dirección Regional de Salud de Tacna detectó altos niveles de contaminación de metales pesados en las aguas que se filtran desde el Embalse de Relaves Quebrada Honda hasta la irrigación Pampa Sitana. Estas aguas son utilizadas para la agricultura y ganadería en la provincia de Jorge Basadre. Así mismo, un informe de la Dirección General de Salud (Digesa) de 2011 reveló que existía un alto grado de concentración de cadmio por encima de los límites permisibles que establecen las normas para el agua residual destinada a fines agrícolas.

Otra controversia ambiental de la SPCC en la región de debe a los humos de la fundición que opera a $15 \mathrm{~km}$ al norte de la ciudad de Ilo. La cual, desde hace más de 40 años descarga al medio ambiente gases sulfurosos y partículas finas de metales pesados por cuatro chimeneas, contaminando la atmósfera del valle de Ilo y provocando lluvia ácida sobre 
suelos y mares, afectando a la población de Ilo, a peces y mariscos y a la agricultura del valle de Ilo y Tambo (Lora, 2015: 9). La fundición emite un promedio de 520 mil toneladas métricas de dióxido de azufre de las cuales 67\% es liberado a la atmósfera (Portocarrero, 2007: 138).

La población del puerto de Ilo ha convivido por décadas con las altas concentraciones de $\mathrm{SO} 2$ en la atmósfera e incluso metales pesados. reportándose una alta tasa de problemas respiratorios (rinitis, faringitis, traqueitis, fibrosis pulmonar, bronquitis crónica y asmatiforme, crisis asmática). La compañía se ha negado a reconocer los daños a la salud de sus operaciones (Lora, 2015: 10). Hecho contrario a lo ocurrido en la fundición del Paso, Texas, donde se cerró la fundición y se ha llevado a cabo un proceso de remediación ambiental (Cunningham \& Tiefenbacher, 2008).

\section{Principales proyectos en exploración de SPCC en Perú}

En total la empresa SPCC tiene 247 títulos de concesión minera en ubicadas en 10 de los 25 departamentos del país (Ver Mapa 2). A continuación, se revisan algunos de los casos más importantes.

\section{a. Departamento de Ayacucho}

En 2009 la empresa adquirió el proyecto minero el Chinchinga, ubicado en el Distrito de Hualla, provincia Víctor Fajardo, Región Ayacucho, con una cantidad efectiva de 6.85 hectáreas. Cerca de las comunidades altoandinas de Hualla y Tiquihua. Que, a finales del 2010, decidieron oponerse a la minería en sus territorios, tanto por temor a la contaminación y agotamiento del agua - pues son comunidades campesinas- como por tener una imagen negativa de la empresa por su accionar en Tacna y Moquegua. A pesar de dicho posicionamiento por parte de la comunidad, "la minera empezó a operar a espaldas de la gran mayoría negociando con un pequeño grupo de dirigentes para obtener una autorización amañada" (Bernaola, 2013:96).

El 15 de julio del 2011 cerca de un millar de comuneros rechazaron los trabajos de la Southern en sus territorios, iniciando una huelga indefinida hasta que se cancelara el proyecto. Posteriormente, en una mesa de diálogo con el gobierno provincial se acordó declarar la intangibilidad de la zona de Chinchinga al ser cabecera de cuenca, así como cancelar todas las concesiones mineras en la provincia Víctor fajardo. Días después, el 21 de Julio la empresa anunció la suspensión del proyecto. 
Sol Pérez-Jiménez. Impacto ambiental de la Compañía minera Southern Perú Cooper Corporation en América Latina: Una aproximación histórica de comienzos del siglo XX a la actualidad

\section{b. Departamento de Arequipa}

El proyecto Tía María ubicado en el Valle del río Tambo ha sido uno de los más controversiales en el Perú. Implica una inversión de US\$ 950 millones de dólares y tendría una duración de 21 años para extraer cobre a través de dos tajos abiertos. La cuestión es que la principal actividad productiva en el valle es la agricultura, por lo que los pobladores se oponen tajantemente a la minería señalando que afectaría la disponibilidad de agua, lo cual limitaría la producción de arroz, caña de azúcar y páprika.

En 2009 las autoridades emitieron la Ordenanza Municipal que convoca a la consulta como mecanismo democrático para recoger el sentido de la población sobre un posible inicio de las actividades mineras. En la cual el $97 \%$ de la población que participo de la consulta se manifestó en contra del proyecto minero. A pesar de la legitimidad de la consulta, las autoridades no sólo la desconocieron, sino que convocaron a una audiencia pública para aprobar el Estudio de Impacto Ambiental (EIA) elaborado por la empresa y avalado por el gobierno.

Lo cual generó varias movilizaciones y paros generales en la región, con una fuerte represión y criminalización por parte de las fuerzas policiales. En marzo de 2011 se inició un paro indefinido y generalizado de la comunidad, que buscaba el rechazo definitivo del EIA del proyecto. La represión de dichas manifestaciones dejo un saldo de 5 muertos y varias decenas de heridos-as. Este hecho sangriento movilizó la opinión pública de todo el país y la opinión internacional, y finalmente el ejecutivo se vio en la obligación de considerar inadmisible el estudio de impacto ambiental.

A fines del año 2012, la empresa volvió a aparecer, señalando que volvería a presentar un nuevo EIA del proyecto Tía María, lo que generó inmediatamente la movilización de la comunidad. A pesar de esto, el 4 de agosto del año 2014, el MINEM aprobó el EIA. Lo cual es inconstitucional pues el proyecto no cuenta con licencia social. A la fecha el proyecto está detenido, el Valle del tambo se continúa movilizando.

\section{c. Departamento de Cajamarca}

Como se mostró las principales actividades de la empresa se encuentran al sur del país. Y, sin embargo, actualmente se plantea explotar también proyectos en el Norte, específicamente, en el Departamento de Cajamarca, cerca de donde años atrás se pretendió desarrollar el proyecto 
minero CONGA. En febrero de 2018 la empresa se adjudicó el proyecto minero Michiquillay, el cual planea comenzar a explotar en 2022. Se trata de uno de los proyectos mineros más grandes impulsados en los últimos años, con una inversión aproximada de US\$2,000 millones. A unos días de adjudicado el proyecto, diferentes comunidades denunciaron que este no debió de haberse aprobado sin la socialización y acuerdos con los sectores Michiquillay, Quinualloc y Pampa Grande. Pues la región Cajamarca tiene grandes problemas con la afectación a los recursos naturales, por lo que se debe ser precavido (RPP Noticias, 2018).

Maspa 2. Departamentos con conecciones mineras de SPCC
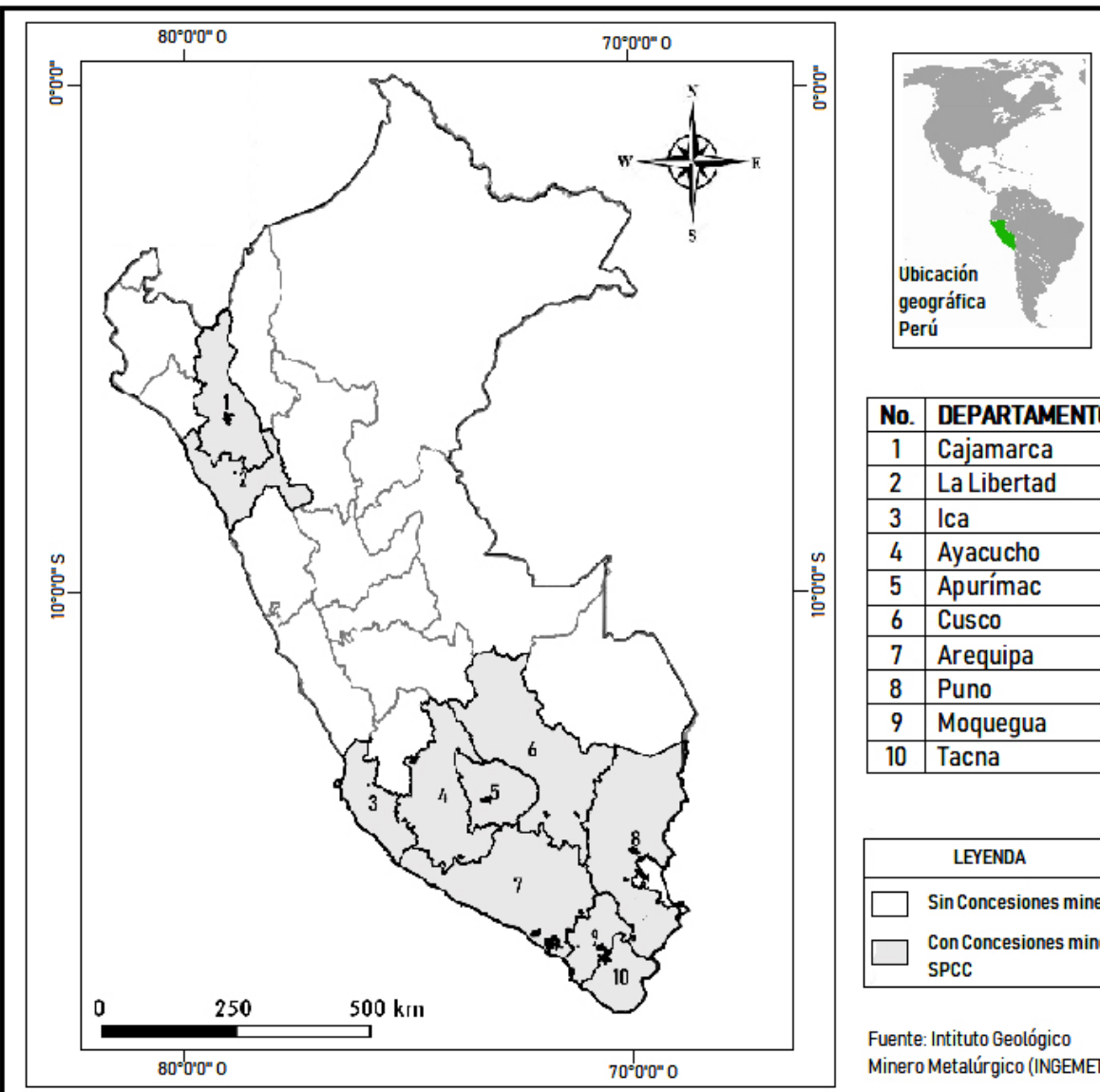

\begin{tabular}{|c|l|}
\hline No. & DEPARTAMENTO \\
\hline 1 & Cajamarca \\
\hline 2 & La Libertad \\
\hline 3 & Ica \\
\hline 4 & Ayacucho \\
\hline 5 & Apurímac \\
\hline 6 & Cusco \\
\hline 7 & Arequipa \\
\hline 8 & Puno \\
\hline 9 & Moquegua \\
\hline 10 & Tacna \\
\hline
\end{tabular}

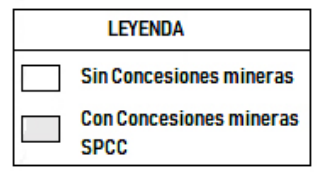

Fuente: Elaboración propia. Con información de SCC (2017). 
Sol Pérez-Jiménez. Impacto ambiental de la Compañía minera Southern Perú Cooper Corporation en América Latina: Una aproximación histórica de comienzos del siglo XX a la actualidad

\section{Despliegue de Southern Peru Cooper Corporation en Latinoamérica}

\section{Ecuador}

La compañía Southern Copper Mediante su filial, Ruta el Cobre SA de CV desarrolla el proyecto cuprífero Chaucha, en la provincia Azuay, el cual se encuentra en fase de exploración avanzada. Southern también tiene participación de 70\% en Minera Ruta de Cobre, en Cuenca. El cual se ha enfrentado a grandes controversias, por encontrarse cerca de una Reserva de la Biosfera llamada el "Macizo de Cajas". Ubicado al suroccidente del Ecuador, en la provincia del Azuay. La cual forma parte de la Red Mundial de Reservas de Biósfera desde el 28 de mayo del 2013, y está integrada por el Programa sobre el Hombre y la Biosfera (MAB) de la UNESCO. Además, dentro del macizo se ubica el Área Nacional de Recreación Quimsacocha, que abarca 3217 hectáreas y en donde nacen los ríos Bermejos y Tarqui, que alimentan a las comunidades de las parroquias de Victoria de Portete y de Tarqui. Por todo ello, el Concejo Cantonal de Cuencas aprobó en enero de 2017, con base a 12 resoluciones oficiales para proteger sus fuentes de agua, una declaratoria que convierte al cantón en un lugar libre de minería. Por dicha razón, el proyecto se encuentra detenido.

\section{Chile}

Southern Copper Corporation llegó a Chile en el año 2000 para iniciar cuatro exploraciones en el norte del país: Catanave, Sierra Áspera, El Salado y Esperanza. De todos los proyectos de exploración, el primero es el que ha presentado más conflictos, principalmente con comunidades Aymaras. Pues se encuentra al interior de la Reserva Nacional de Las Vicuñas, que forma parte del Sistema Nacional de Áreas Silvestres Protegidas por el Estado (SNASPE).

En marzo del año 2011, la alcaldía de Putre se resistió a la aprobación del proyecto, presentando un recurso de protección ante la Corte de Apelaciones, asegurando que la empresa había infringido normativas medioambientales y de protección a las comunidades Aymaras cercanas. Por su parte, las mismas comunidades indígenas afectadas presentaron otro recurso argumentando la falta de consulta. Sin embargo, ambas peticiones fueron rechazadas argumentando que no hubo acto ilegal o arbitrario. 
Sol Pérez-Jiménez. Environmental impact of the Southern Peru Copper Corporation Mining Company in Latin America: A historical approach from the Early 20th Century to the present

Mapa 3. Países en los que opera SPCC en Latinoamérica

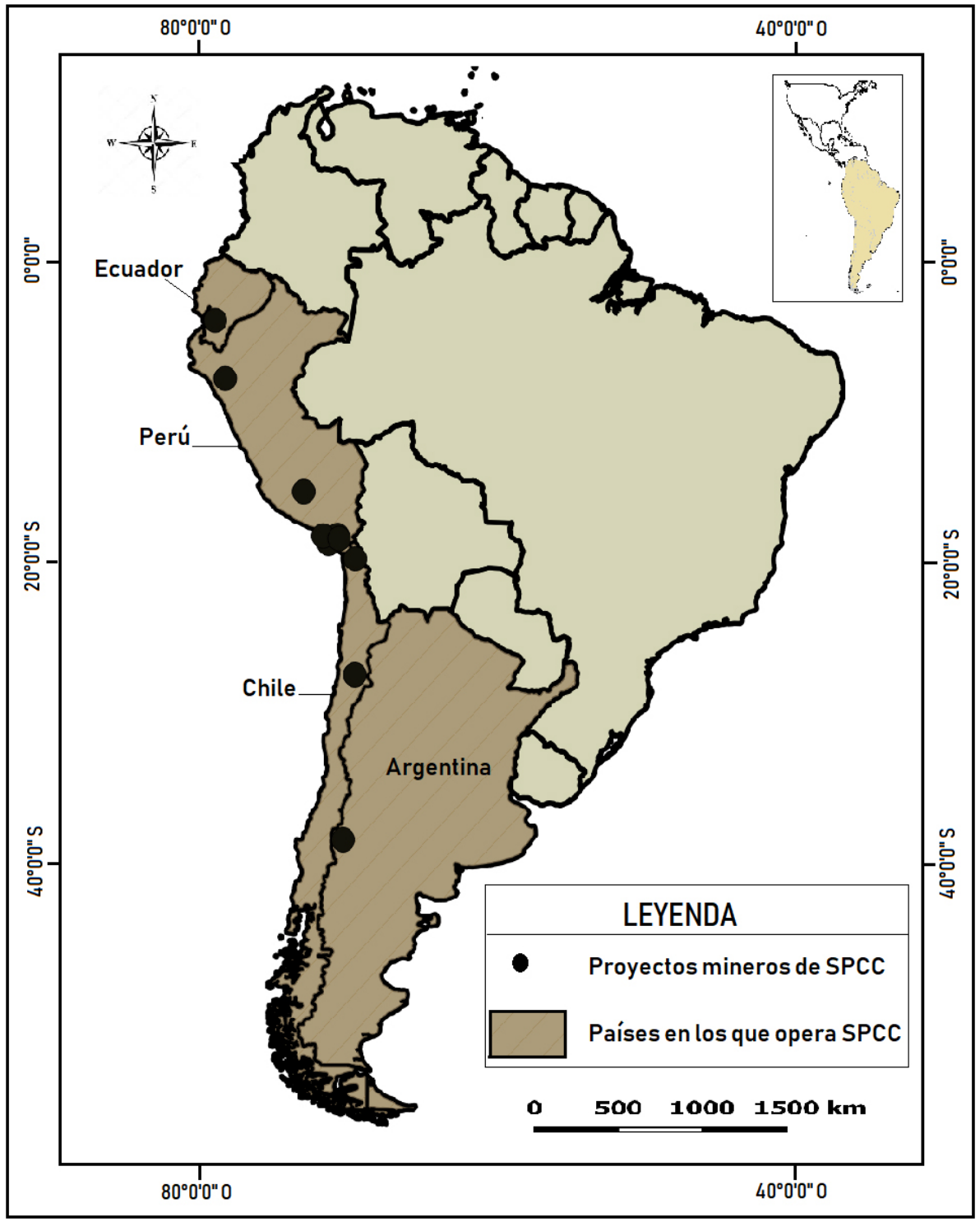

Fuente: Elaboración propia. 
Sol Pérez-Jiménez. Impacto ambiental de la Compañía minera Southern Perú Cooper Corporation en América Latina: Una aproximación histórica de comienzos del siglo XX a la actualidad

\section{Argentina}

La empresa tiene una filial denominada Southern Copper Argentina mediante la cual desarrolla un proyecto de exploración de cobre en la provincia patagónica de Neuquén. Donde pretende desarrollar los proyectos de exploración Las Nenas y La Voluntad. Una de las mayores preocupaciones sobre este proyecto minero es la afectación de la cuenca del río Catán Lil, fuente de agua para la población local. En 2015 se formó un grupo de vecinos auto convocados para resistir el emprendimiento. Asimismo, comunidades Mapuche se oponen al proyecto, pues la minera se quiere instalar en territorio sagrado mapuche. Las cuales denuncian que "no se realizó ningún tipo de consulta de este plan, que pone en riesgo la vida no solo de las comunidades mapuche sino también de toda la población que vive en los márgenes de los ríos afectados y sus zonas de influencia" (Servindi, 2016).

\section{Conclusiones}

La compañía minera Grupo México y sus subsidiarias, Asarco y Southern Cooper Corporation han presentado desde sus inicios, en su afán de lucro y acumulación, un patrón de uso irracional de recursos, como el agua, y de abstención o abandono de sus responsabilidades de protección ambiental y derechos de las comunidades. Tan es así que varios proyectos de la compañía se encuentran tanto en áreas de conservación ambiental, como territorios indígenas. El desempeño de la compañía en sus minas de Toquepala y Cuajone, y la emergencia del conflicto Tía María, SPCC ha dado como resultado que las comunidades donde la compañía pretende operar se oponen a esta.

Finalmente, es necesario reflexionar si, estamos dispuestos a seguir contaminando y agotando recursos estratégicos en beneficio de corporaciones privadas y en detrimento de una gran cantidad de poblaciones afectadas en el continente latinoamericano. Especialmente en un contexto de escases de recursos y cambio climático como el que atravesamos en la actualidad. 


\section{Referencias}

Arciniega, R. (1986). Los mineros de la Southern Peru Copper Corporation, 1968-1981. Pontificia Universidad Católica del Perú, Facultad de Ciencias Sociales. Lima, Perú.

Aste, J. \& Portugal, M. (1984). Las perspectivas en la refinación del cobre en el Perú. Fundación Friedrich Eber-ECO. Lima

Bernaola, D. (2013). El caso del proyecto de exploración minera Chinchinga, Ayacucho. En: Quiñones, P. \& Bernaola, D. (Coords.). 2013. Los límites de la expansión minera en Peru. Asociación servicios educativos rurales, SER, Lima, Perú.

Contraloría General de la República (1990). Examen especial sobre la contaminación ambiental producida por la actividad minero-metalúrgica en la zona sur del país (valles de Ilo y Tambo, Ciudad de Ilo y Bahía de Ite). Ed. Contraloría General de la República, Lima, Perú. Cunningham, C. A., \& Tiefenbacher, J. P. (2008). Evaluating the effectiveness of public participation efforts by environmental agencies: repermitting a smelter in El Paso, Texas, USA. Environment and Planning C: Government and Policy, 26(4), 841-856.

Diaz Palacios, J. (1988). El Perú y su medio ambiente (No. T01 D5).

Dictamen de la Comisión Bicameral Investigadora del Contrato de Toquepala, Lima (1977). Informe CEDAL, Lima, 1982.

Hoetmer, R., Castro, M., Daza, M., De Echave, J. C., \& Ruiz, C. (2013). Minería y movimientos sociales en el Perú. Instrumentos y propuestas para la defensa de la vida, el agua y los territorios. Lima: Programa democracia y transformación global, CooperAcción, Acsur, Entrepueblos.

Informe periodístico. (2015). Décadas de operación en Moquegua y Tacna generan desconfianza Labor "Contaminación por metales pesados Tóxicos producidos por las operaciones Minero Metalúrgicas de la SPCC".

Lora, J. (2015). Trasnacionales mineras y ecocidio en el Perú. Disponible en: http://rcci.net/globalizacion/2004/fg471.htm

Malpica, C. (1976). Los dueños del Perú. Ed. Peisa. Lima, Perú.

Observatorio de Conflictos Mineros de América Latina (OCMAL). (2015). Irrupción SPCC en Territorio Aymara. Disponible en: http://www. conflictosmineros.net/contenidos/9-chile/5254-minera-southerncopper-corporation-irrumpe-en-territorio-aymara 
Sol Pérez-Jiménez. Impacto ambiental de la Compañía minera Southern Perú Cooper Corporation en América Latina: Una aproximación histórica de comienzos del siglo XX a la actualidad

Oviedo, D. (2014). Medidas de mitigación ambiental de la Bahía de Ite. Programa de Adecuación y Manejo Ambiental. Perú: Southern Copper Southern Perú (SPCC). Recuperado de https://es.slideshare.net/ ogdturtacna/medidas-de-mitigacin-ambiental-en-la-baha-de-ite

Pajuelo, R., Farfán, C. \& Asencios, D. (2012). Documento de trabajo No.851. Cronología del Conflicto Social Perú 2011. Publicación electrónica, Consejo Latinoamericano de Ciencias Sociales (CLACSO). En Observatorio Social de América Latina (OSAL), 29, 4 -12.

Portocarrero, S., Sanborn, C. \& Camacho, L. A. (2007). Moviendo montañas: empresas, comunidades y ONG en las industrias extractivas. Perú: Universidad del Pacífico.

Sánchez, F. (1981). Minería, capital trasnacional y poder en el Perú, CEPRODE, Lima.

Servicios de Comunicación Intercultural (Servindi). (2016). Argentina: Mapuches en alerta por proyecto minero de Southern Copper. Recuperado de https://www.servindi.org/print/56516

Southern Copper Corporation (SCC). (2017). Informe Anual. Southern Cooper Corporation. 
